\title{
O bservation sur simulateur des stratégies de contrôle de vannes de ralenti actionnées par moteurs pas à pas
}

\author{
V. Mahieu ${ }^{1}$, B. Leduc ${ }^{1}$ \\ 1 Service de mécanique appliquée, Université libre de Bruxelles, avenue F.D. Roosevelt 50, CP 165/41, B-1050 Bruxelles - Belgique \\ e-mail : vmahieu@ulb.ac.be
}

Résumé - Le contrôle moteur par calculateur électronique peut être résumé par un échange de signaux provenant de capteurs, envoyés au calculateur, qui déduit et calcule à partir de grandeurs cartographiées, les instructions de commande envoyées aux actionneurs.

L'article présenté relate l'observation de stratégies de pilotage d'une vanne de ralenti actionnée par moteur pas à pas. La vanne est raccordée à son calculateur d'origine, émulé par un simulateur pour systèmes de contrôle moteur. Les stratégies observées concernent une vanne de ralenti dont le pointeau est actionné linéairement, afin de moduler le débit d'air de contournement (by-pass).

Divers modèles (modèle de démarrage, modèle de simulation routière) permettent d'observer les stratégies de contrôle du ralenti par ce type de vanne, et de retrouver certaines calibrations.

Mots-clés : contrôle moteur par l'électronique, simulateur moteur, stratégies de contrôle du ralenti.

\begin{abstract}
Observation of Idle Regulation Strategies using Stepper Motors Idle Valves - Engine control by ECU (Electronic Control Unit) can be shortly described by informations coming from sensors sent to the ECU, and by calculated and setting values sent to actuators by the ECU.

This paper describes idle regulations observations on an engine simulator. The observation of specific idle driving strategies is especially performed by using an ECU which drives an idle valve based on stepper motor linear actuator.

Various sub-models like starting models or road models allow idle pintle motion patterns to be generated, showing idle control strategies and calibrations.

Keywords: electronic engine control, engine simulator, idle control strategies and calibrations.
\end{abstract}




\section{IN TRO DUCTION}

Le contrôle moteur par calculateur électronique peut être résumé par un échange de signaux, provenant de capteurs et envoyés au calculateur, qui déduit et calcule à partir de grandeurs cartographiées, les instructions de commande envoyées aux actionneurs (fig. 1) [1-3].

L'article présenté relate l'observation de stratégies de pilotage d'une vanne de ralenti actionnée par moteur pas à pas. La vanne est raccordée à son calculateur d'origine, émulé par un simulateur pour systèmes de contrôle moteur [4].

Le simulateur développé crée un environnement moteur virtuel, permettant d'activer, en vue de leur étude, différents systèmes d'injection électronique.

En pratique (fig. 2), les signaux moteurs sont recréés artificiellement à l'aide d'un générateur de signaux. Ce dernier, piloté par un PC, permet non seulement de simuler le fonctionnement normal d'un moteur, mais également d'explorer des plages de fonctionnement extrême, voire physiquement impossible pour le moteur (par exemple, très faibles et très hauts régimes).

Ensuite, les réactions du calculateur sont observées par l'intermédiaire du même PC. On retrouve à l'écran les différents paramètres sous forme graphique ou numérique. Un outil, appelé modèle découplé, permet de scanner les informations contenues dans les mémoires du calculateur et de reconstruire certaines cartographies.

Les stratégies observées concernent une vanne de ralenti dont le pointeau est actionné linéairement, afin de moduler le débit d'air de contournement, ou by-pass (fig. 3) [6].

Un potentiomètre linéaire est monté solidaire du mouvement du pointeau, et fait remonter l'information vers le PC (fig. 4).

Il faut noter que la régulation du ralenti est assurée par deux paramètres de contrôle, que sont l'avance à l'allumage (action rapide) et le débit d'air additionnel dosé par la vanne de ralenti (action lente) [2]. Nous nous sommes limités ici à décrire le comportement de la vanne d'air additionnel.

\section{EXPLO ITATION DU MO DĖLE DE DÉMARRAGE}

En réalisant une montée en régime exponentielle de 0 à 1000 tr/min, par pas de 20 tours, ce modèle permet de simuler le comportement du moteur pendant sa phase de démarrage et d'observer les stratégies de commande d'injecteurs, d'avance à l'allumage et d'ouverture de la vanne d'air additionnel.

Les exemples qui suivent illustrent les résultats que l'on peut retirer de ce modèle de transitoire de démarrage. L'influence de paramètres tels que la température $\left(T^{\circ}\right)$ de liquide de refroidissement ou l'ouverture papillon y est facilement mise en évidence.

Avant d'interpréter les résultats, il est important de rappeler que ce modèle opère en boucle ouverte : la vitesse de rotation du moteur (tr/min) suit une progression programmée, non influencée par les réactions des paramètres censés la contrôler (avance à l'allumage et vannage d'air additionnel).

\section{Démarrage à chaud : comparaison avec et sans ouverture papillon}

Les figures 5 et 6 donnent l'évolution des grandeurs suivantes, en fonction du temps :

- la commande électrique de la vanne (V) ;

- la vitesse d'ouverture de la vanne (pas/s);

- le pourcentage d'ouverture de la vanne (\%);

- le régime moteur (tr/min).

L'essai montre que la vanne se prépositionne à $20 \%$ d'ouverture (valeur cartographiée) et maintient cette position jusqu'à environ $500 \mathrm{tr} / \mathrm{min}$.

Ensuite, le régime augmentant, et la consigne de $850 \mathrm{tr} / \mathrm{min}$ n'étant pas atteinte (voir les correspondances dans le tableau 1), la vanne s'ouvre progressivement, en ralentissant au fur et à mesure que l'on s'approche de la consigne. Une fois la consigne franchie, le régime se stabilise rapidement à $1000 \mathrm{tr} / \mathrm{min}$, ce qui conduit à une fermeture à vitesse constante de la vanne (écart à la consigne constant).

Lorsque le papillon des gaz est ouvert à $50 \%$, le comportement est beaucoup plus simple à décrire : étant donné que le moteur reçoit déjà suffisamment d'air par la tranche papillon, la vanne se prépositionne à $8 \%$, et se referme complètement une fois la consigne atteinte.

Le chapitre suivant est consacré à l'exploitation du modèle découplé.

\section{EXPLOITATION DU MODÈLE DÉCOUPLÉ}

Le modèle découplé présente l'avantage de pouvoir faire varier indépendamment les unes des autres toutes les grandeurs d'entrée du calculateur.

En pratique, on retrouve à l'écran du PC les curseurs permettant de fixer :

- le régime moteur (tr/min);

- la pression collecteur $(\mathrm{hPa})$;

- l'ouverture papillon $(\%)$;

- la température de liquide de refroidissement $\left({ }^{\circ} \mathrm{C}\right)$;

- la température d'air à l'admission $\left({ }^{\circ} \mathrm{C}\right)$;

- la fréquence du signal de sonde $\lambda(\mathrm{Hz})$.

Dans le cas présent, le modèle découplé permet d'observer l'influence du régime, de la charge et de la température du liquide de refroidissement sur les stratégies d'ouverture de la vanne d'air additionnel.

\subsection{Régulation du ralenti : influence d'une variation de régime (Delta N)}

L'exemple repris en figure 7 illustre la réaction de la vanne d'air pour un moteur ayant atteint son régime thermique. $\mathrm{La}$ température du liquide de refroidissement vaut alors $100^{\circ} \mathrm{C}$. 


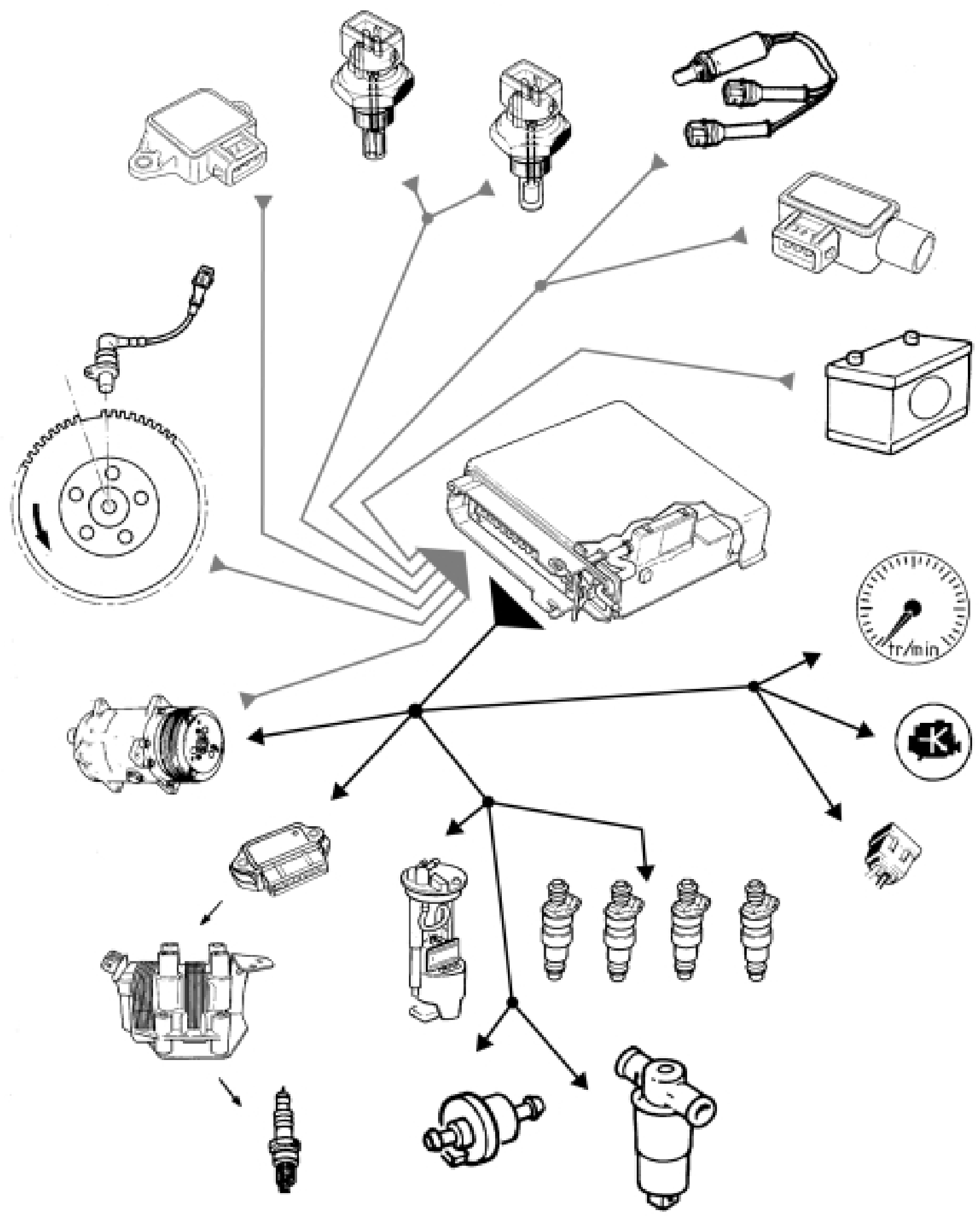

Figure 1

Principes du contrôle moteur (note technique PSA TU3J2).

Engine control basics (technical note PSA TU3J2). 

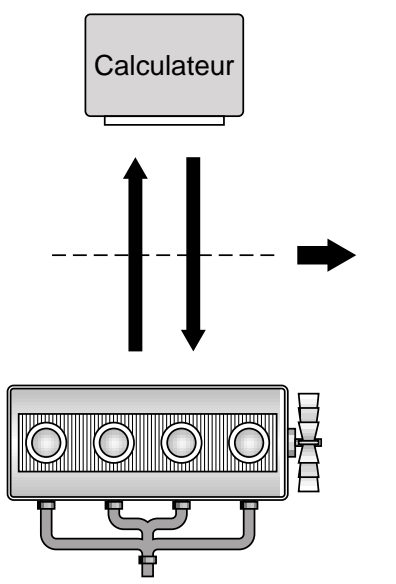

Figure 2

Substitution du moteur par le simulateur.

Engine substitution.

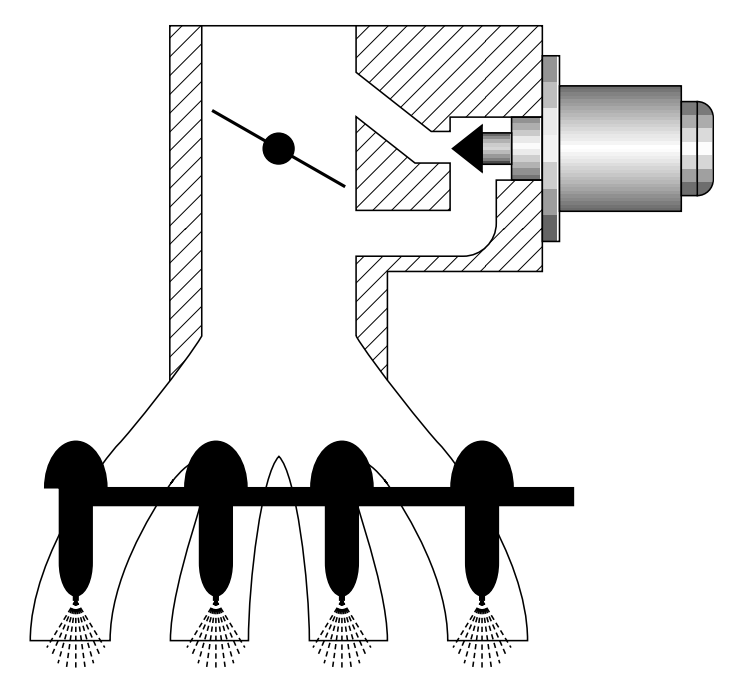

Figure 3

By-pass de contournement.

Idle valve on bypass duct.

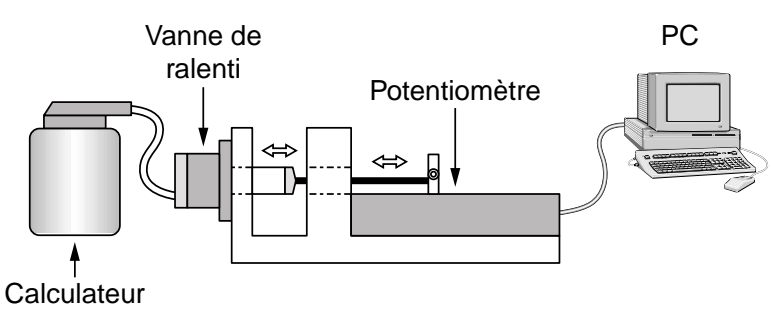

Figure 4

Pointeau équipé d'un potentiomètre.

Valve pintle position sensor.

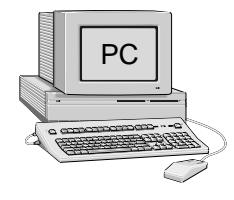

La consigne de régime pour une telle température se situe aux alentours de $850 \mathrm{tr} / \mathrm{min}$. L'essai consiste à générer différents écarts de régime autour de la consigne :

$$
\text { Delta } N=\operatorname{ABS}\left(N_{\text {réel }}-N_{\text {consigne }}\right)
$$

Le régime de consigne $N_{\text {consigne }}$ est une fonction de la température de liquide de refroidissement, comme repris dans le tableau 1.

TABLEAU 1

$N_{\text {consigne }}$ fonction de $T^{\circ}$ liq rpm $_{\text {target }}$ function of $T_{\text {liq }}^{\circ}$

\begin{tabular}{c|c}
\hline$T_{\text {liq }}^{\circ}\left({ }^{\circ} \mathrm{C}\right)$ & $\sim N_{\text {consigne }}(\operatorname{tr} / \mathrm{min})$ \\
0 & 1150 \\
20 & 1100 \\
40 & 1000 \\
60 & 950 \\
80 & 900 \\
100 & 850 \\
120 & 850 \\
\hline
\end{tabular}

Le relevé des paramètres commence sous un régime de $500 \mathrm{tr} / \mathrm{min}$, bien inférieur au régime de consigne.

Naturellement, la vanne se positionne très ouverte, à $80 \%$ d'ouverture (valeur de réglage cartographiée en mémoire du calculateur).

Ensuite, le régime passe à $1000 \mathrm{tr} / \mathrm{min}$. La vanne engage une fermeture lente, caractérisée ici par une faible pente.

Si l'on monte encore le régime à $1200 \mathrm{tr} / \mathrm{min}$, la pente de fermeture s'accentue, mettant en évidence le lien entre vitesse de fermeture et écart à la consigne de régime.

Un coefficient de proportionnalité est introduit, reliant la vitesse d'ouverture à Delta $N$ :

Vitesse d'ouverture vanne $(\mathrm{pas} / \mathrm{s})=K \cdot$ Delta $N(\mathrm{tr} / \mathrm{min})$

$K$ est normalement exprimé en $\frac{\mathrm{pas} / \mathrm{s}}{\mathrm{tr} / \mathrm{min}}$.

Les relevés expérimentaux donnent pour l'ouverture, avec $K$ rapporté à un écart de 100 tr/min :

$$
\mathrm{K}_{\text {ouv }}=4,1\left(\frac{\mathrm{pas} / \mathrm{s}}{100 \mathrm{tr} / \mathrm{min}}\right)
$$

et pour la fermeture :

$$
K_{\text {ferm }}=8,8\left(\frac{\mathrm{pas} / \mathrm{s}}{100 \mathrm{tr} / \mathrm{min}}\right)
$$

Ceci permet de conclure que, pour un même écart à la consigne, la vanne se ferme deux fois plus vite qu'elle ne s'ouvre. 

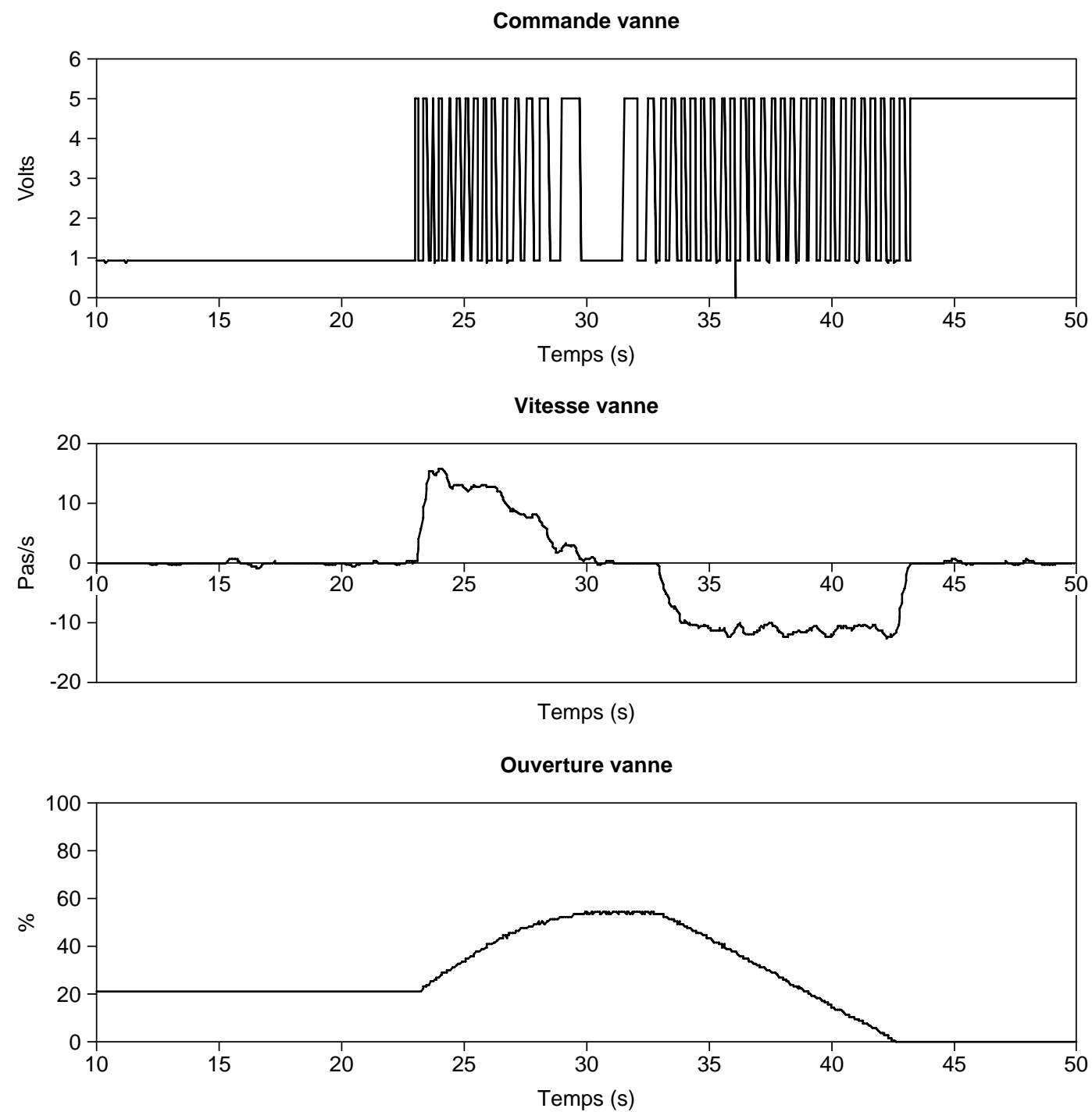

Régime moteur

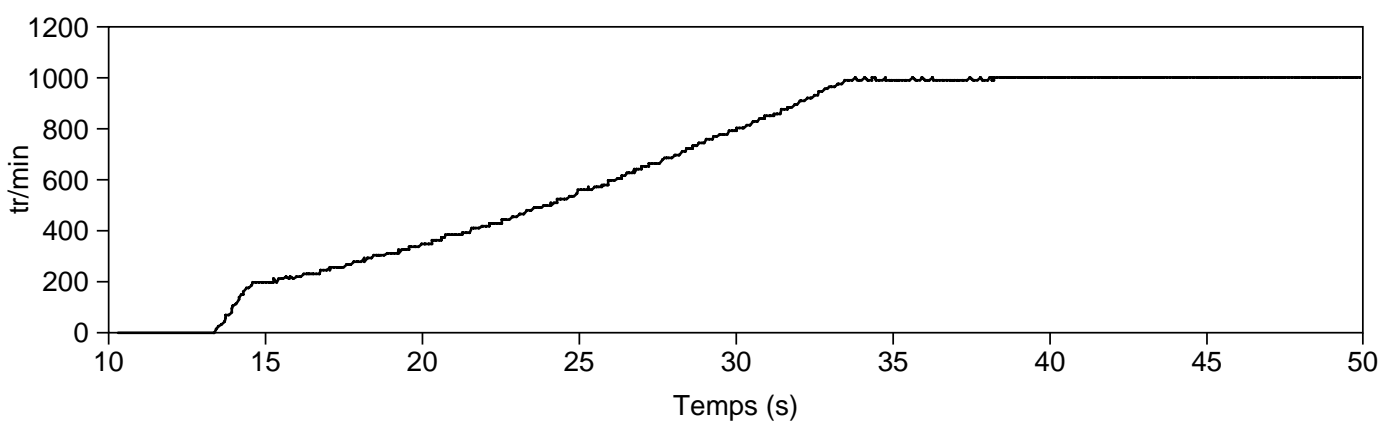

\begin{tabular}{|l|l|}
\hline Tension batterie $=13,5 \mathrm{~V}$ & $T^{\circ}$ air $=20^{\circ} \mathrm{C}$ \\
\hline Fréq. sonde $=10 \mathrm{~Hz}$ & $T^{\circ}{ }_{\text {liq }}=100^{\circ} \mathrm{C}$ \\
\hline Pression coll. $=324 \mathrm{hPa}$ & Ouv. papillon $=0 \%$ \\
\hline
\end{tabular}

Figure 5

Démarrage sans ouverture papillon.

Starting with no load. 


\section{Commande vanne}

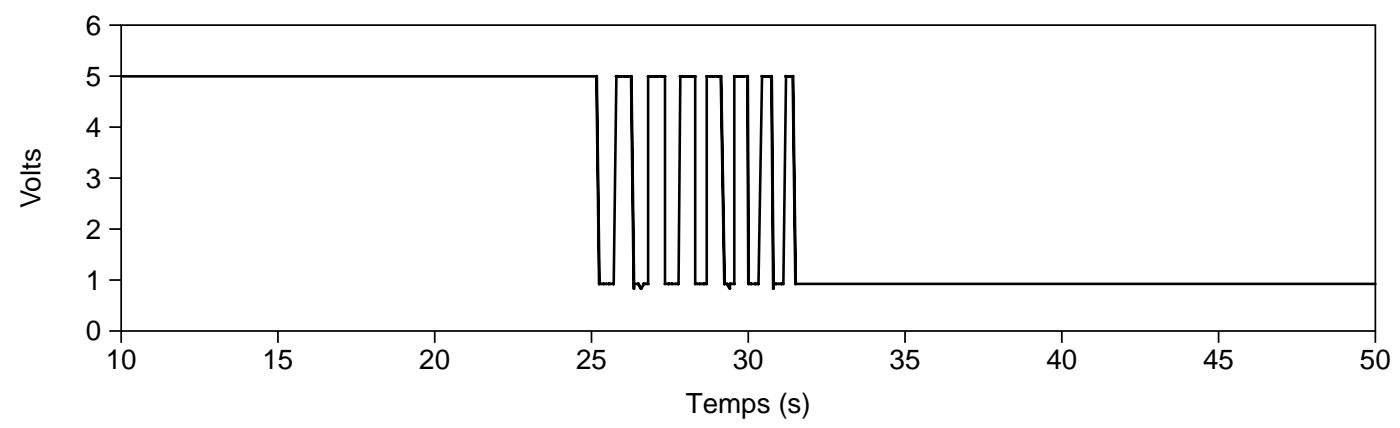

Vitesse vanne

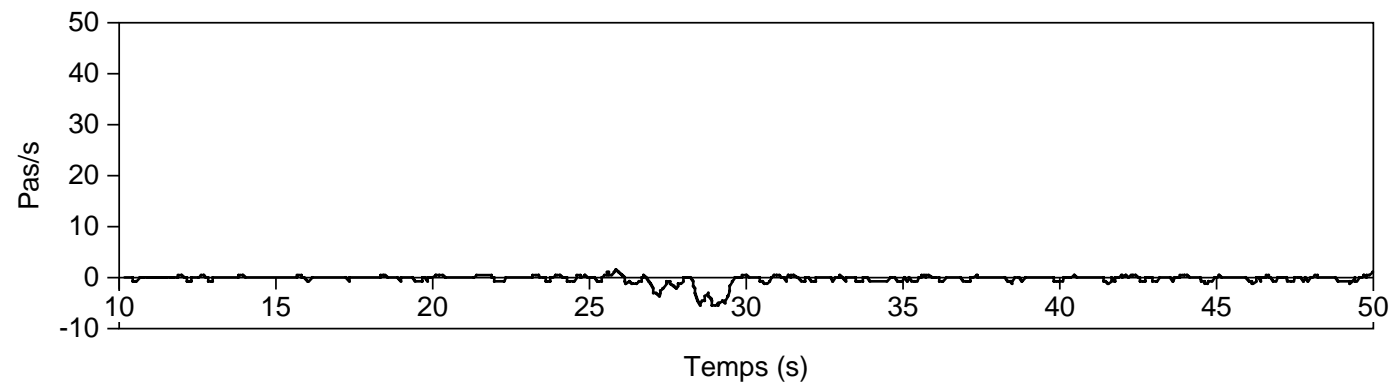

Ouverture vanne

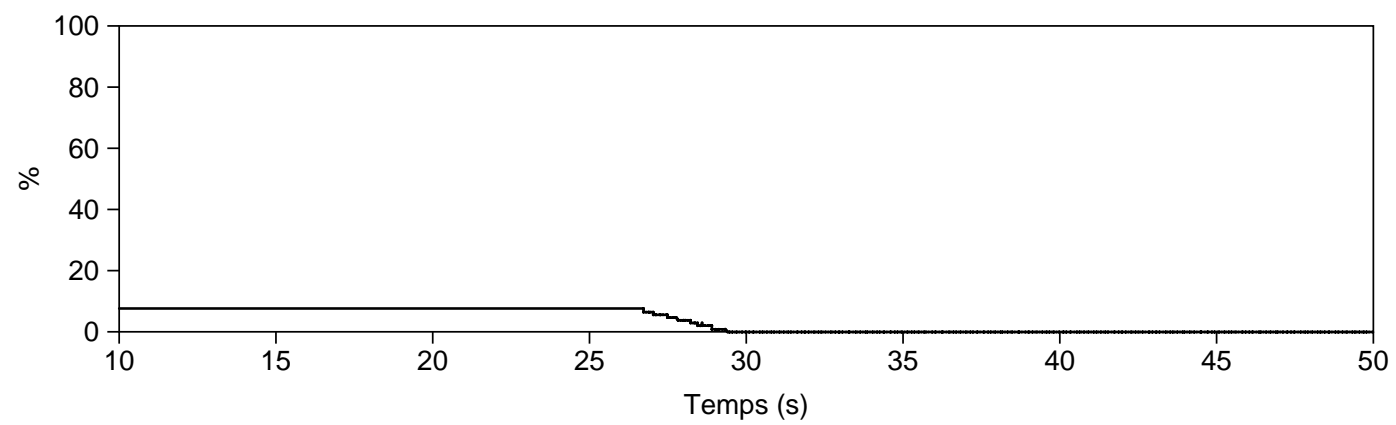

Régime moteur

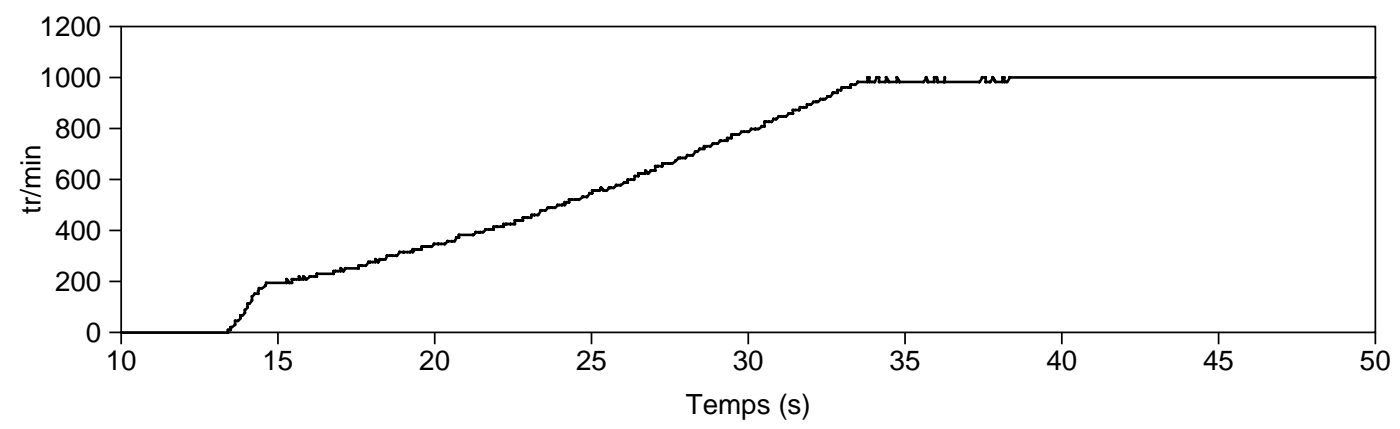

\begin{tabular}{|l|l|}
\hline Tension batterie $=13,5 \mathrm{~V}$ & $T^{\circ}{ }_{\text {air }}=20^{\circ} \mathrm{C}$ \\
\hline Fréq. sonde $=10 \mathrm{~Hz}$ & $T^{\circ}{ }_{\text {liq }}=100^{\circ} \mathrm{C}$ \\
\hline Pression coll. $=324 \mathrm{hPa}$ & Ouv. papillon $=50 \%$ \\
\hline
\end{tabular}

Figure 6

Démarrage avec ouverture $50 \%$.

Starting with load. 

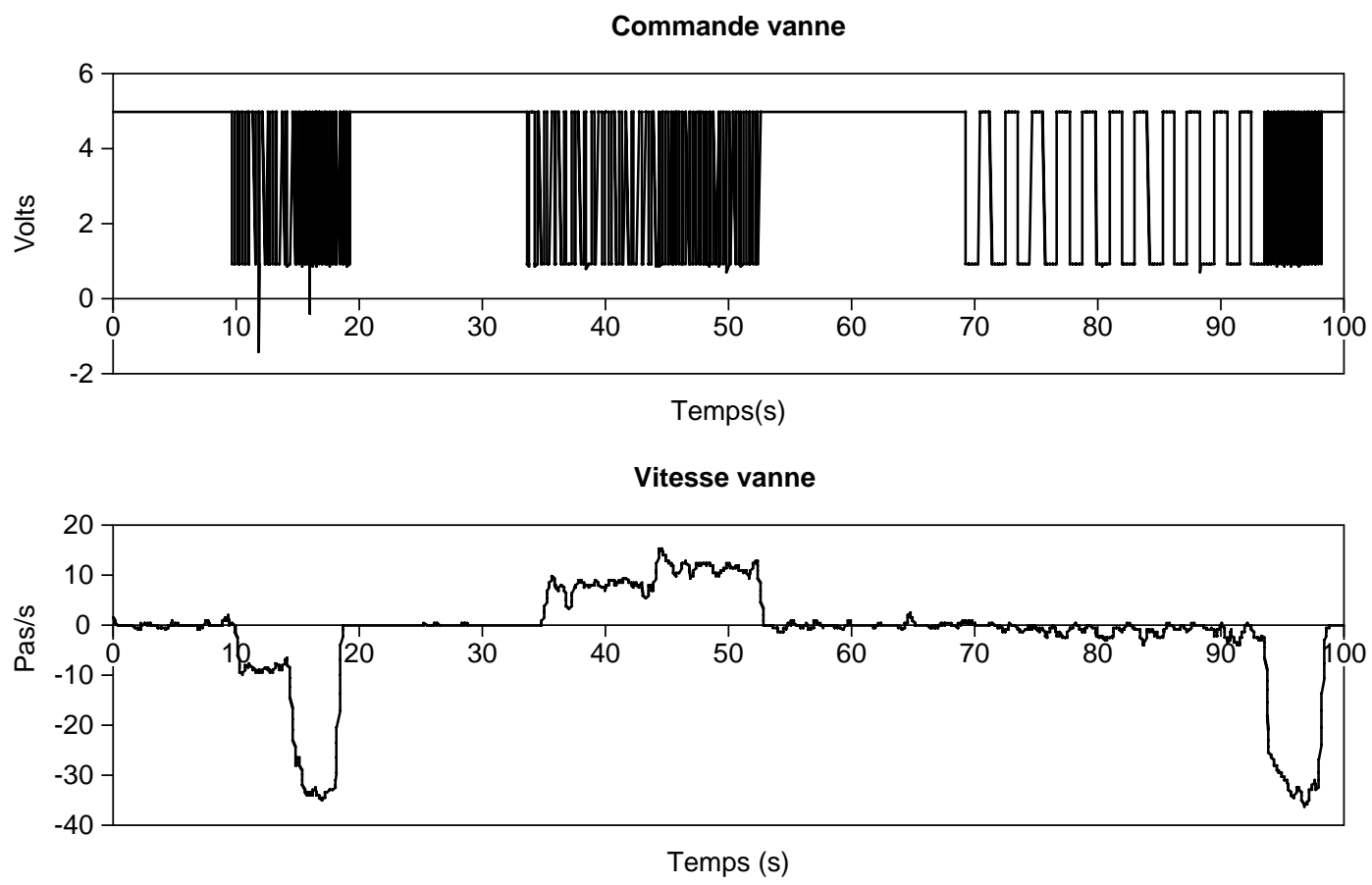

Ouverture vanne

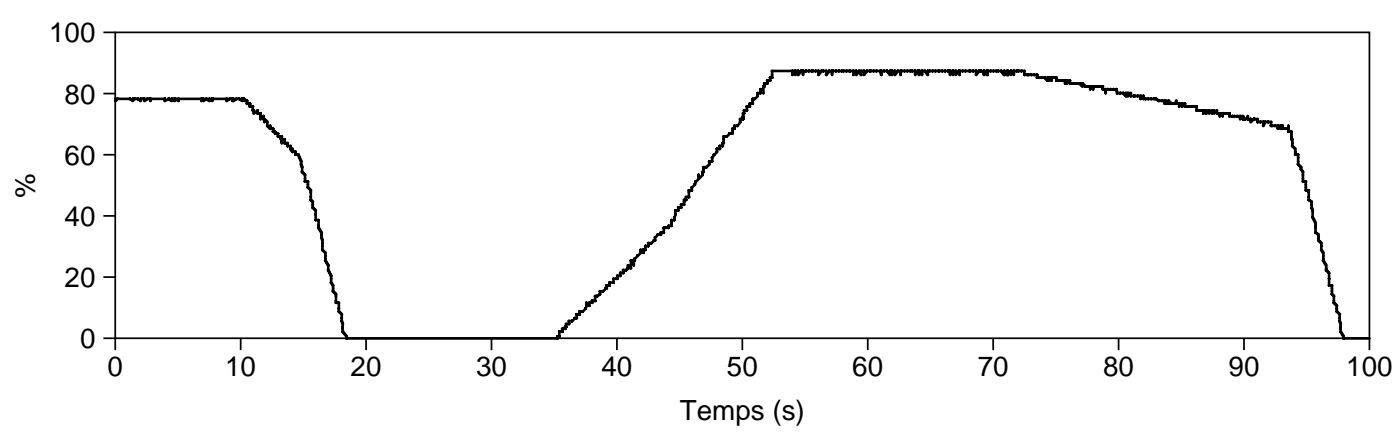

Régime moteur

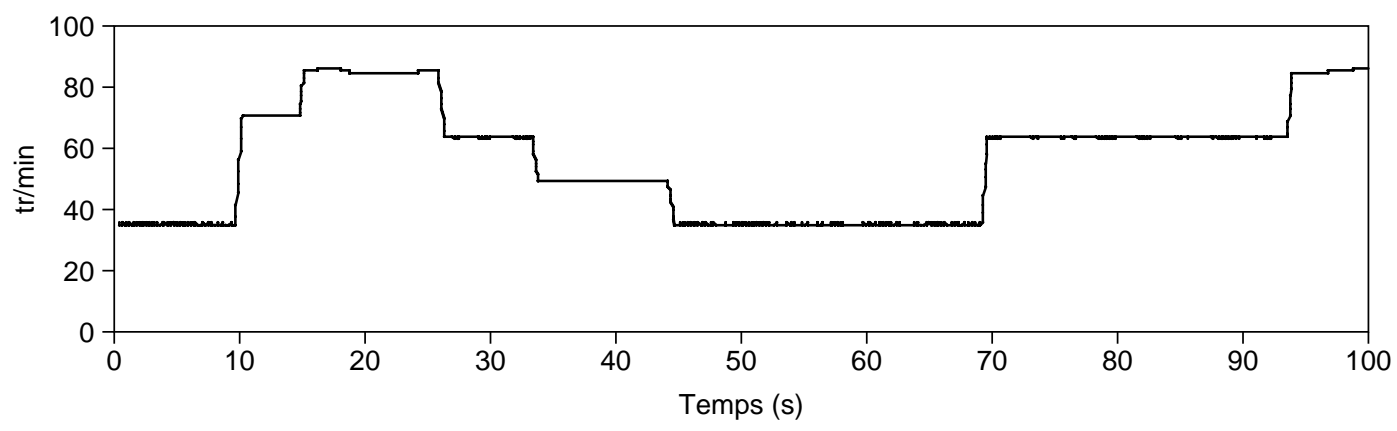

\begin{tabular}{|l|l|}
\hline Tension batterie $=13,5 \mathrm{~V}$ & $T^{\circ}$ air $=20^{\circ} \mathrm{C}$ \\
\hline Fréq. sonde $=10 \mathrm{~Hz}$ & $T^{\circ}$ liq $=100^{\circ} \mathrm{C}$ \\
\hline Pression coll. $=324 \mathrm{hPa}$ & Ouv. papillon $=0 \%$ \\
\hline
\end{tabular}

Figure 7

Influence de Delta $N$ sur la régulation.

Delta $N$ influence on regulation. 

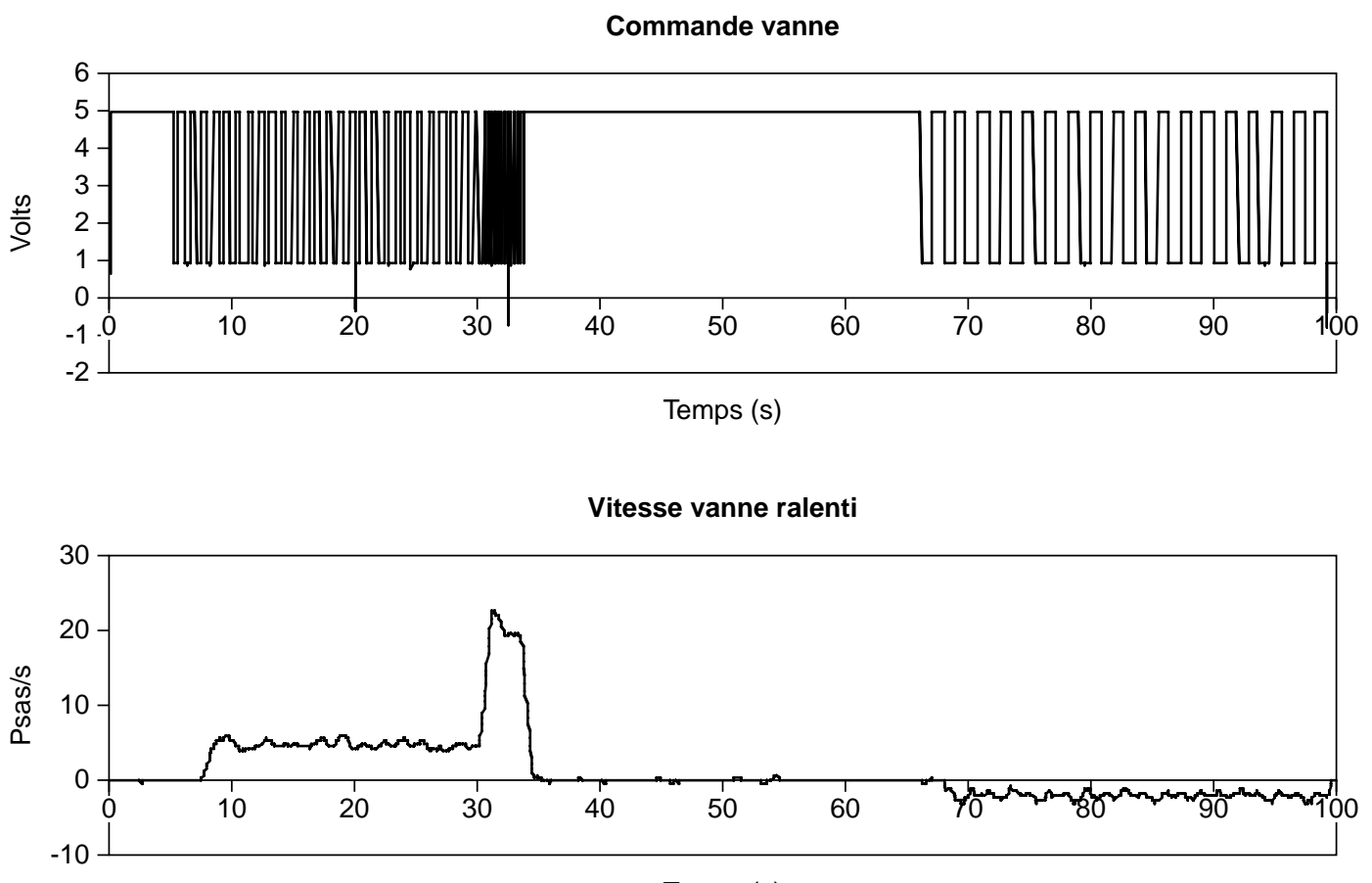

Temps (s)
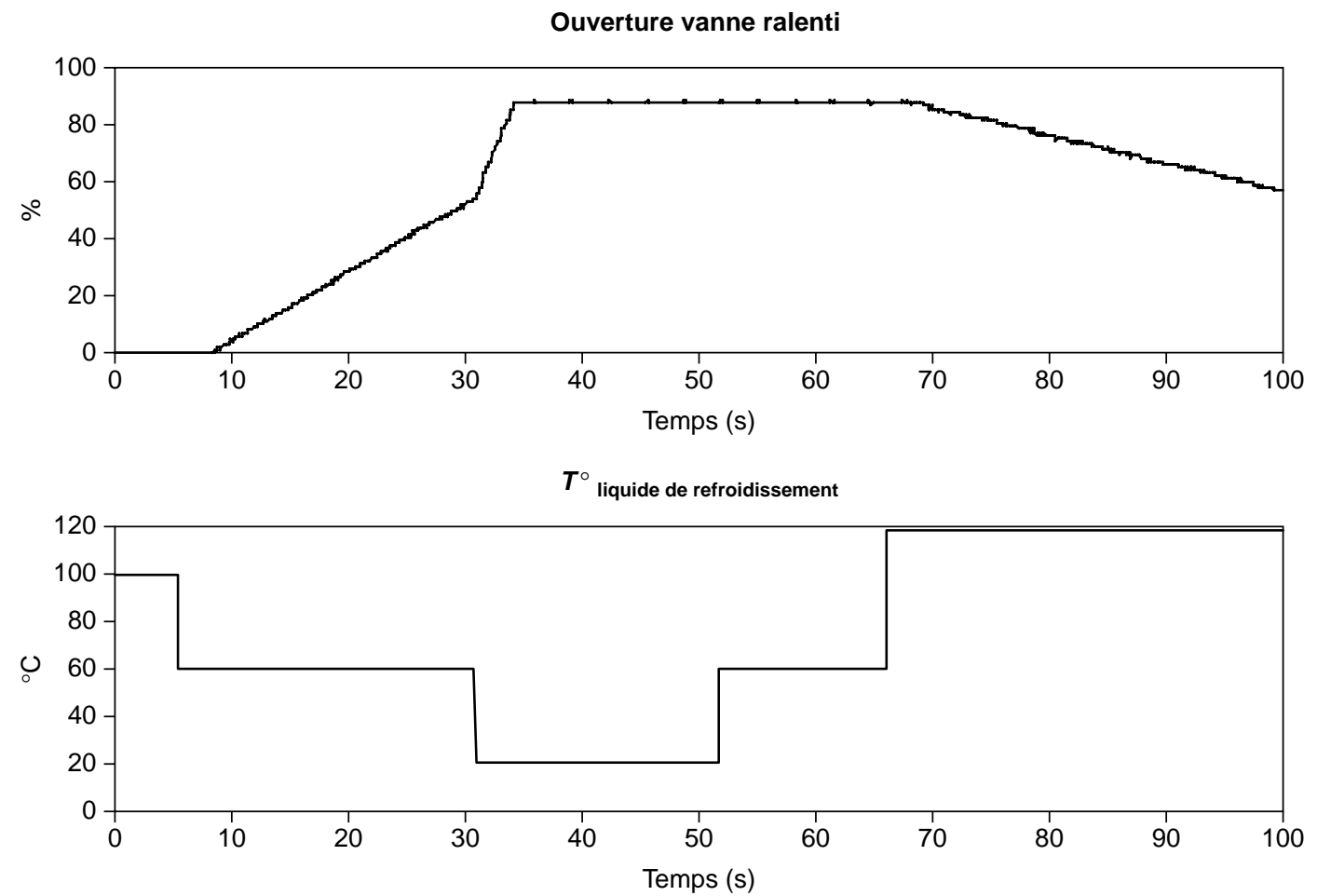

\begin{tabular}{|l|l|}
\hline Tension batterie $=13,5 \mathrm{~V}$ & $T^{\circ}{ }_{\text {air }}=0^{\circ} \mathrm{C}$ \\
\hline Fréq. sonde $=10 \mathrm{~Hz}$ & $N=900 \mathrm{tr} / \mathrm{min}$ \\
\hline Pression coll. $=324 \mathrm{hPa}$ & Ouv. papillon $=0 \%$ \\
\hline
\end{tabular}

Figure 8

Influence de Delta $T^{\circ}$.

Delta $T^{\circ}$ influence on regulation. 

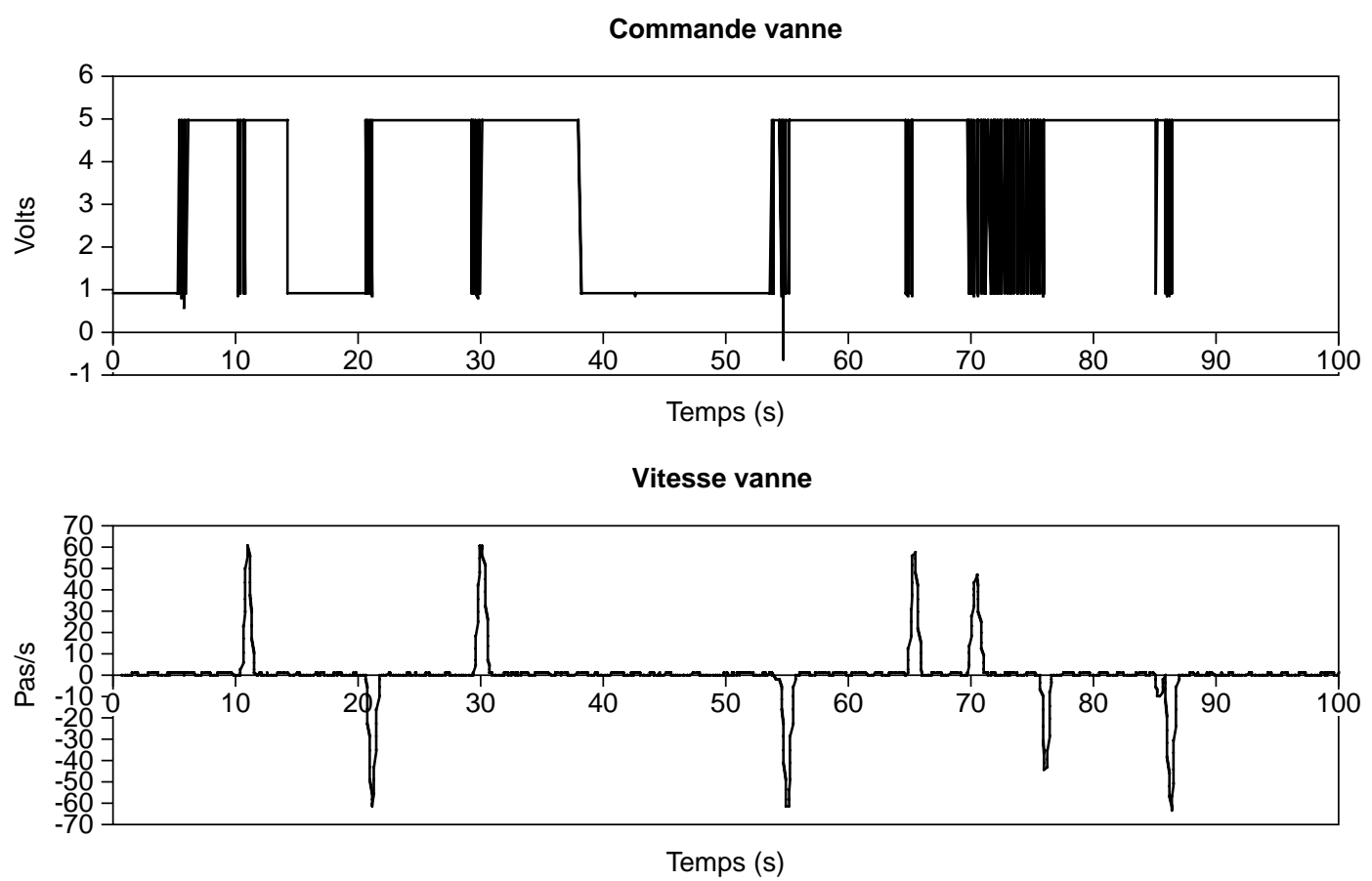

Ouverture vanne

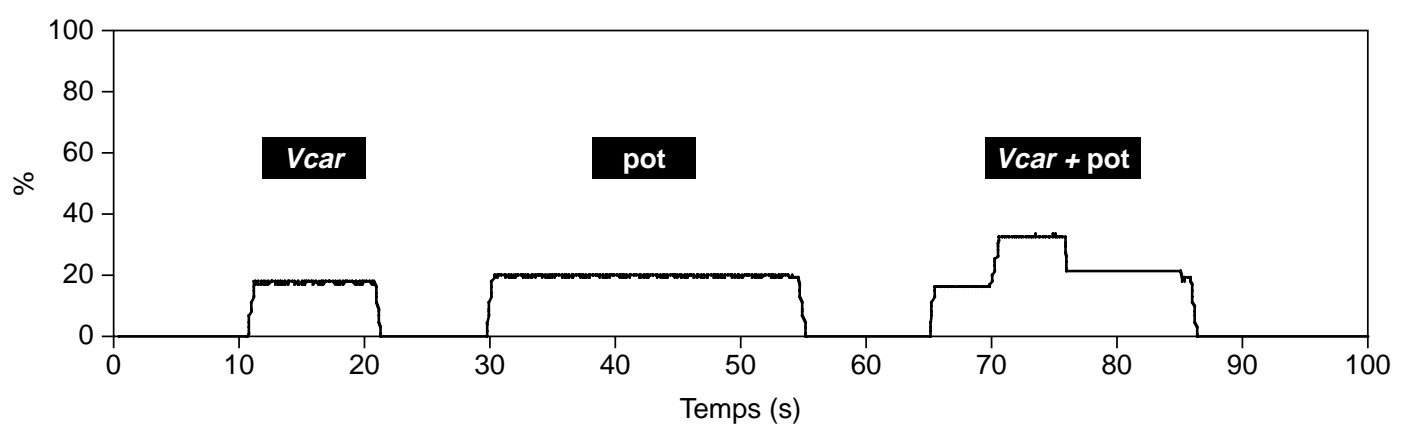

Ouverture papillon

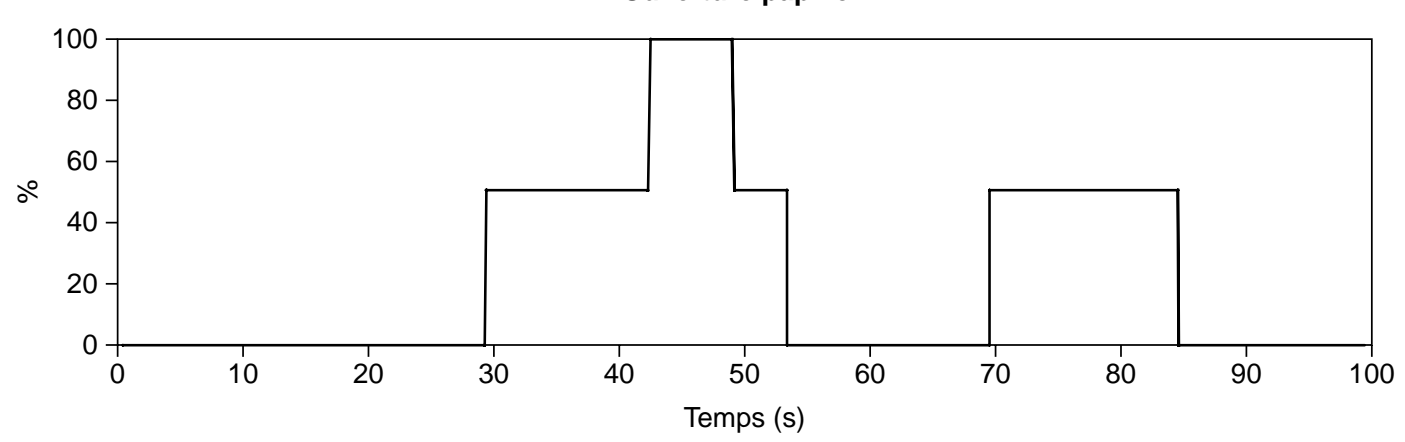

\begin{tabular}{|l|l|}
\hline Tension batterie $=13,5 \mathrm{~V}$ & $T^{\circ}{ }_{\text {air }}=20^{\circ} \mathrm{C}$ \\
\hline Fréq. sonde $=10 \mathrm{~Hz}$ & $T^{\circ}{ }_{\text {liq }}=0^{\circ} \mathrm{C}$ \\
\hline Pression coll. $=324 \mathrm{hPa}$ & $N=1500 \mathrm{tr} / \mathrm{min}$ \\
\hline
\end{tabular}

Figure 9

Influence de l'ouverture papillon et de la vitesse véhicule.

Delta load and Vcar influence on regulation. 

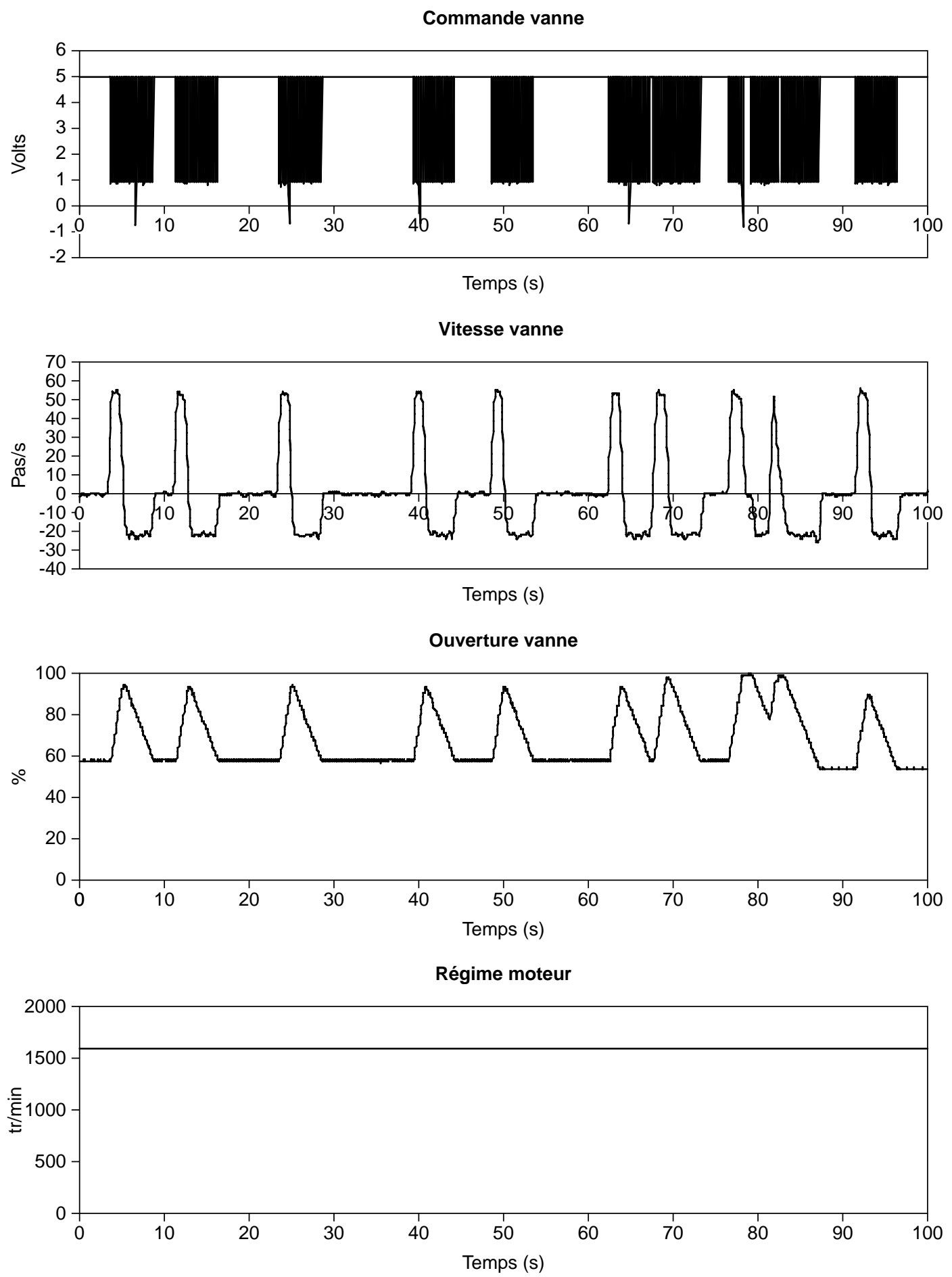

\begin{tabular}{|l|l|}
\hline Tension batterie $=13,5 \mathrm{~V}$ & $T^{\circ}{ }_{\text {air }}=0^{\circ} \mathrm{C}$ \\
\hline Fréq. sonde $=10 \mathrm{~Hz}$ & $T^{\circ}{ }_{\text {liq }}=0^{\circ} \mathrm{C}$ \\
\hline Pression coll. $=324 \mathrm{hPa}$ & Ouv. papillon $=0 \%$ \\
\hline
\end{tabular}

Figure 10

Comportement instable de la vanne.

Unsteady valve behaviour. 

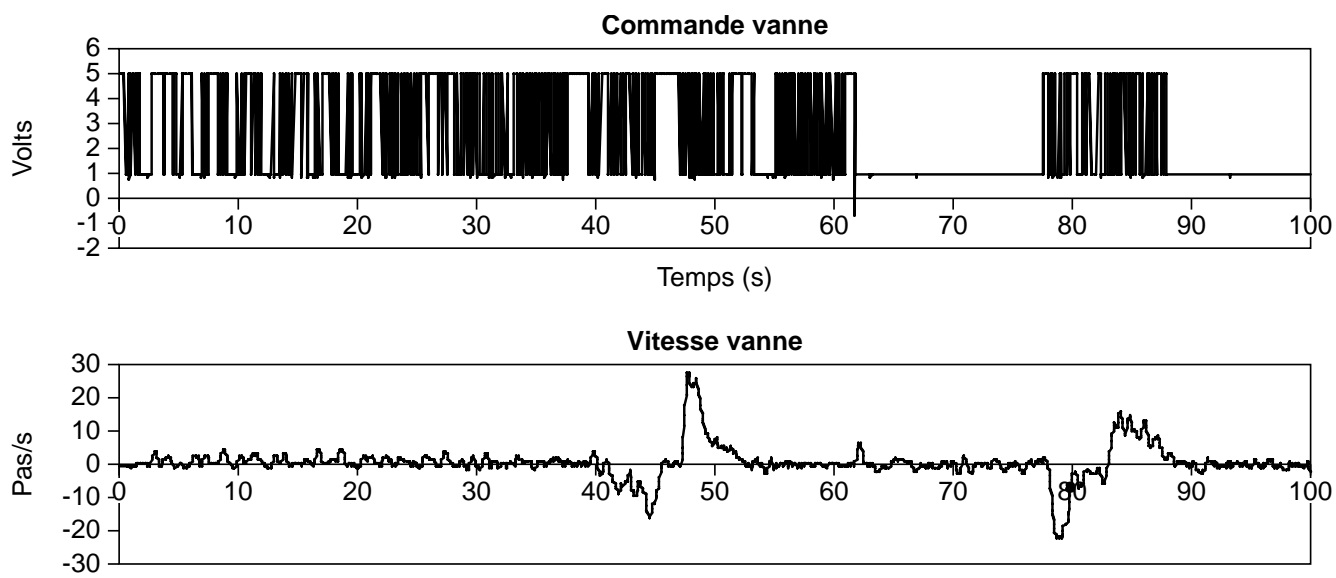

Temps (s)
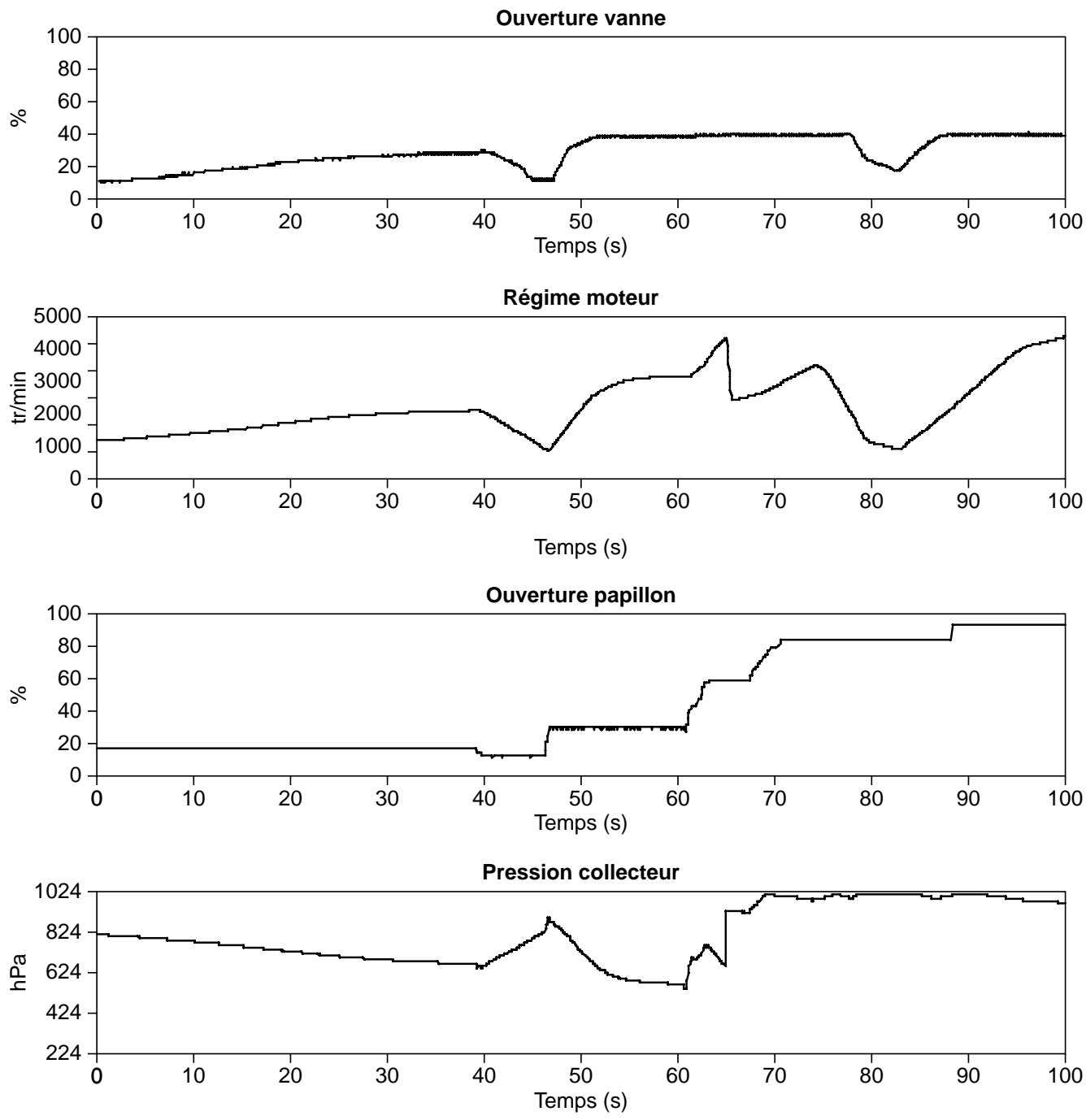

\begin{tabular}{|l|l|}
\hline Tension batterie $=13,5 \mathrm{~V}$ & $T^{\circ}{ }_{\text {air }}=20^{\circ} \mathrm{C}$ \\
\hline Fréq. Sonde $=10 \mathrm{~Hz}$ & $T^{\circ}$ liq $=90^{\circ} \mathrm{C}$ \\
\hline
\end{tabular}

Figure 11

Réaction de la vanne en transitoire.

Transient idle valve response. 


\subsection{Régulation du ralenti : influence de la température de liquide de refroidissement}

Ce test est effectué à vitesse de rotation constante, égale à $900 \mathrm{tr} / \mathrm{min}$, correspondant à une température de liquide de refroidissement égale à $80^{\circ} \mathrm{C}$.

En jouant sur cette température, on influe sur la consigne de régime de ralenti.

Pour une température basse, $900 \mathrm{tr} / \mathrm{min}$ est une valeur trop faible, et pour une température suffisamment élevée, $900 \mathrm{tr} / \mathrm{min}$ est un régime trop élevé.

L'observation semble donc la même, si ce n'est que dans la configuration de cet essai, la fermeture de la vanne est deux fois plus lente que l'ouverture.

Ce changement de stratégie peut éventuellement être expliqué par le fait que le système de contrôle du moteur s'attend à gérer de rapides variations du régime moteur (régulation classique), mais que de brusques et rapides variations de la température du liquide de refroidissement peuvent apparaître comme des anomalies de fonctionnement, et conduire à une stratégie de secours (mode dégradé).

Ce test est illustré en figure 8.

\subsection{Régulation du ralenti : influence de l'ouverture papillon et de la vitesse véhicule}

La progression d'un véhicule au sein d'un embouteillage présente une situation relativement complexe à gérer pour le système chargé de réguler le ralenti. En plus des transitoires de démarrage et d'arrêt véhicule, il faut également distinguer si le ralenti est à stabiliser au point mort (véhicule à l'arrêt), ou avec le rapport de première vitesse engagé (véhicule roulant au pas).

Ce cas de figure est simulé sur la figure 9. Le régime moteur est maintenu constant à $1500 \mathrm{tr} / \mathrm{min}$. Le moteur étant chaud, la vanne tente de ramener le régime vers $900 \mathrm{tr} / \mathrm{min}$, en se positionnant fermée.

Si l'on envoie un signal proportionnel à la vitesse de rotation de la roue du véhicule au calculateur (Vcar sur la figure 9), quel qu'en soit le niveau, la vanne de ralenti se positionne à $20 \%$ d'ouverture, pour tenir compte de la charge additionnelle due à la mise en mouvement du véhicule.

Le signal provenant du potentiomètre lié au papillon et signalant un enfoncement de la pédale des gaz est également pris en compte par le calculateur. Ce dernier impose une ouverture de la vanne à $20 \%$ (trou à l'accélération + dashpot, décrit ci-après). La combinaison des deux effets donne une ouverture de $40 \%$.

Cette gestion de l'alimentation en air du moteur cherche à combiner le traditionnel compromis entre émissions réduites et agrément de conduite.

\subsection{Régulation du ralenti : comportement instationnaire}

Le simulateur est capable de générer des points de fonctionnement en dehors des limites habituelles.

Pour l'exemple donné en figure 10 , le régime est fixé à $1600 \mathrm{tr} / \mathrm{min}$. Le moteur est froid, sans charge ni ouverture papillon. De ce fait, le régime de ralenti choisi à 1600 tr/min est anormalement élevé.

Un comportement instable de la vanne est observé : en effet, partant d'une ouverture élevée, à $60 \%$, la vanne tente de reprendre le contrôle du ralenti, dans une rapide succession d'ouvertures et de fermetures.

Notons que dans ce cas, la fermeture est deux fois plus lente que l'ouverture.

L'étape suivante est consacrée à l'observation de la vanne à l'aide d'un modèle de comportement routier (temps réel) [6].

\section{MODĖLE DE SIMULATION ROUTIÈRE}

Le modèle routier génère des phases de fonctionnement représentatives d'accélérations et de décélérations réelles.

Les paramètres pris en compte par le modèle sont :

- les spécifications du véhicule (cartographies du couple et de la pression collecteur, rapports de boîte et de pont, masse totale, Cx, etc.) ;

- les instructions du conducteur (ouverture papillon et rapport de boîte engagé) ;

- le profil de la route.

La pente de la route reste fixée à $0 \%$ pour cet essai.

Sur ce transitoire (fig. 11), qui combine des variations d'ouverture papillon, de régime et de rapport de boîte, il apparaît clairement que l'ouverture de la vanne suit l'évolution du régime moteur.

Au-dessus de 3000 tr/min, la vanne reste ouverte à $40 \%$. En dessous, elle se referme progressivement, jusqu'à se stabiliser à $15 \%$.

Cette stratégie porte le nom de fonction dashpot.

De cette façon, la vanne anticipe toute fermeture rapide du papillon suite à un levé de pied du conducteur. Elle se tient prête à en tempérer les effets, en adoucissant la chute de débit d'air par une fermeture progressive. De la sorte, de trop grands écarts de richesse (néfastes pour le rendement du catalyseur) sont évités, et cela contribue également à l'agrément de conduite, en appliquant progressivement la mise en frein moteur.

\section{CON CLUSION S, LMITES ET PERSPECTIVES}

Cette étude présente l'exploitation d'un simulateur pour calculateur. Ce simulateur s'avère être un moyen efficace d'observation des stratégies et des calibrations du contrôle moteur. 
Par ailleurs, des comportements particuliers ont été relevés, concernant :

- le prépositionnement de la vanne ;

- le transitoire de démarrage ;

- le rôle de l'écart à la consigne de ralenti ;

- l'influence de l'ouverture papillon ;

- l'influence de la vitesse véhicule ;

- la fonction dashpot.

L'outil développé présente cependant un certain nombre de limitations.

Les modèles opèrent en effet en boucle ouverte, sans la moindre réaction vis-à-vis des grandeurs que le calculateur cherche à réguler.

Ensuite, la version actuelle est dédiée à un type de calculateur. Le passage à une autre version ou à une autre gamme concurrente n'est pas très souple, et demande une révision profonde des câblages et calibrations du générateur de signaux.

Afin d'y remédier, les développements ultérieurs concerneront un nouveau simulateur, plus polyvalent et modulaire, capable d'émuler les nouvelles générations de calculateurs de contrôle moteur, incluant le réglage de la charge par papillon motorisé.

Les études se focaliseront alors sur les stratégies de contrôle de ces papillons motorisés (ETC: Electronic Throttle Control).

\section{RÉFÉRENCES}

1 Bosch, R. (1990) Électricité et électronique pour l'automobile à moteur à essence, Delta Press France.

2 Kjergaard, L., Nielsen, S. et Hendricks, S. (1994) Advanced Nonlinear Engine Idle Speed Control System. SAE, article 940974.

3 (1996) Electronic Engine Controls. SAE, SP 1149.

4 Neuman, D. (1994) Universeller Echtzeitsimulator zur Generierung von Motorensignalen. MTZ Motortechnische Zeitschrift, 55, article 12.

5 Mahieu, V., Geels, P.Y. (1996) Idle Valve Pintle Design by Numerical Flow Simulation. SAE, article 960038.

6 Moskwa, J. (1995) Automotive Engine Modeling for RealTime Control using MATLAB/Simulink. SAE, article 950417.

Manuscrit définitif reçu en décembre 1999 